\title{
CALPHAD-Based Integrated Computational Materials Engineering Research for Materials Genomic Design
}

\author{
WEI XIONG ${ }^{1,2}$ \\ 1.-Department of Materials Science and Engineering, Northwestern University, Evanston, \\ IL 60208, USA. 2.—e-mail: wxiong@yahoo.com
}

Due to materials genomic databases created by versatile computational tools in thermodynamics and kinetics, CALPHAD (CALculation of PHAse Diagrams) is one of the most important techniques in materials and manufacturing design. The CALPHAD method is neither empirical nor fundamental, but it plays a vital role to bridge engineering and science, industry and academia. ${ }^{1,2}$ Evidently, CALPHAD-related models and experiments have been extensively adopted in materials and process design for both alloys and oxides. ${ }^{2-4}$ There are three components in the materials innovation infrastructure of the Materials Genome Initiative (MGI) strategy: digital data, computational, and experimental tools. Such an idea can be well reflected in the framework of the modern CALPHAD research. As indicated in Fig. 1, modern CALPHAD modeling is based not only on experimental data but also on the combination of data with atomistic modeling, typically, quantum mechanical calculations. The integration of such techniques further extends spatial and temporal ranges of CALPHAD models. In this way, CALPHAD and its developed databases can be used in other simulation techniques for designing material properties by controlling phases and microstructure evolutions. It should be noted that experiments provide actual data resources for validating and calibrating multiscale simulation and modeling, which is indispensable in the modern CALPHAD infrastructure. Moreover, the amount of experimental needs is inversely proportional to the quality and accuracy of the computational models. Overall, the CALPHAD methodology can leverage various computational techniques to construct the integrated computational materials engineering (ICME) system design tools for materials genomic research.

Wei Xiong is the guest editor for the Alloy Phases Committee of the TMS Functional Materials Division and TMS Structural Materials Division, and is the coordinator of the topic CALPHAD-Based ICME Research for Materials Genomic Design in this issue.
For the TMS 2015 Annual Meeting \& Exhibition, 41 research papers were received for the dedicated MGI symposium, "CALPHAD-Based ICME Research for Materials Genomic Design," sponsored by the Alloy Phases Committee and the Integrated Computational Materials Engineering Committee. Contributed talks covered (I) materials genomic design in engineering applications, (II) CALPHAD database construction and materials genomic data repository, (III) advanced processing and characterization in materials design, and (IV) CALPHADbased ICME model and tool development.

Indeed, it is a formidable task for this JOM topic to represent the entire CALPHAD-based ICME research for materials genomic design. However, one may start learning the whole through seeing a part of it presented in this $J O M$ issue. Furthermore, we hope that the MGI symposium during the TMS conference along with these papers housed in the current issue can stimulate more discussions on CALPHAD-based ICME research for materials genomic design.

In the current special topic of JOM, three papers are contributed:

- T.N. Bhat, L.M. Bartolo, U.R. Kattner, C.E. Campbell, and J.T. Elliott: "Strategy for Extensible, Evolving Terminology for the Materials Genome Initiative Efforts": Without common rules and standards, database and simulation tools will not be able to develop in a sustainable way. National Institute of Science and Technology (NIST) has initiated considerable efforts on open CALPHAD database and materials data repository. ${ }^{5}$ This paper proposes a rules-based approach with initial examples from a growing corpus of materials terms in the NIST Materials Data Repository, which is limited not to the CALPHAD community but to the entire community contributing to Materials Genome.

- S. Chen, W. Cao, F. Zhang, Q. Li, J. Zhang, C. Zhang, and J. Zhu: "Calculation of 2-Dimension 


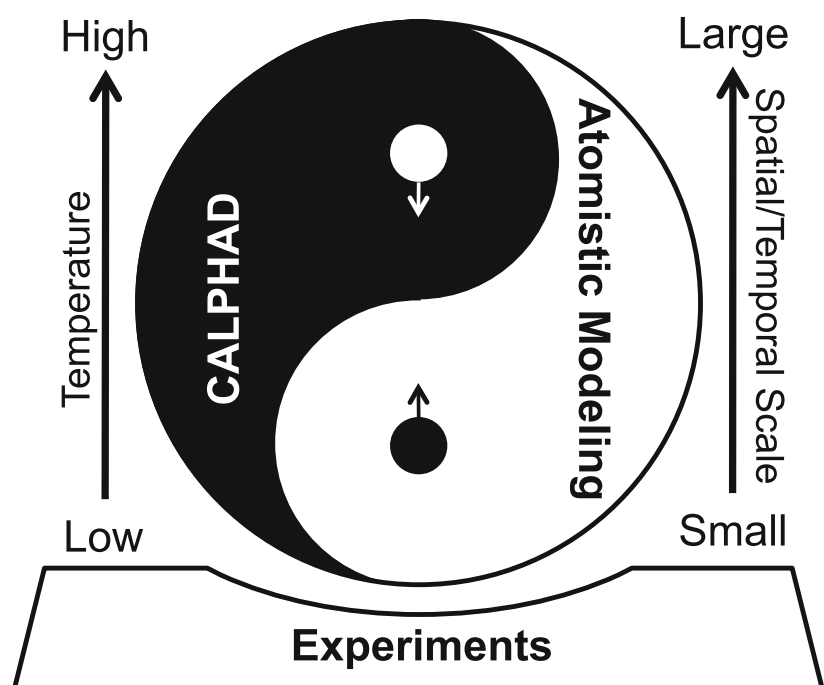

Fig. 1. Illustration of modern concept of the CALPHAD methodology. Experiments provide data resources for supporting both atomistic modeling and CALPHAD. Circles with arrow ends shown in the middle of the Taiji diagram represent spins for challenging issues on magnetic transition. ${ }^{6,7}$.

and 3-Dimension Phase Diagrams": Successful materials design relies on robust models and effective software. Developments of related phase diagram calculation tools with both twodimensional and three-dimensional visualizations have been made using the concept of the zero-phase fraction and one-phase fraction, respectively. This will facilitate both materials design research and education in fundamentals of materials science.
- J. Zhu, C. Zhang, W. Cao, S. Chen, and F. Zhang: "Molar Volume Modeling of Ti-Al-Nb and Ti-AlMo Ternary Systems": Using the CALPHAD method, the thermodynamics of both $\mathrm{Ti}-\mathrm{Al}-\mathrm{Nb}$ and $\mathrm{Ti}-\mathrm{Al}-\mathrm{Mo}$ systems have been optimized with the thermodynamic description of molar volume, which can be used for alloy density prediction during the materials design. This paper might trigger more thoughts and discussion on constructing materials database of various thermodynamic properties using the CALPHAD methodology.

These papers being published under the topic of CALPHAD-Based ICME Research for Materials Genomic Design provide excellent details and research on the subject. To download any of the papers, follow the URL http://link.springer.com/ journal/11837/67/8/page/ 1 to the table of contents page for the August 2015 issue (vol. 67, no. 8).

\section{REFERENCES}

1. L. Kaufman and J. Ågren, Scripta Mater. 70, 3 (2014).

2. N. Saunders and A.P. Miodownik, CALPHAD Calculation of Phase Diagrams A Comprehensive Guide, vol. 1 (Oxford: Pergamon, 1998).

3. G.B. Olson and C.J. Kuehmann, Scripta Mater. 70, 25 (2014).

4. U.R. Kattner, JOM 49, 14 (1997)

5. C.E. Campbell, U.R. Kattner, and Z.K. Liu, Scripta Mater. 70, 7 (2014).

6. W. Xiong, Q. Chen, P. Korzhavyi, and M. Selleby, $C A L$ PHAD 39, 11 (2012).

7. A.I. Lichtenstein, M.I. Katsnelson, and G. Kotliar, Phys. Rev. Lett. 87(6), 672051 (2001). 